CLAWAR 2018: 21st International Conference on Climbing and Walking Robots and the Support Technologies for Mobile Machines, Panama City, Panama, 10-12 September 2018

\title{
BIO-INSPIRED QUADRUPED ROBOT FOR DETECTION CARBON DIOXIDE IN THE AIR
}

\author{
MARIA CAMILA ROJAS SUÁREZ, SANTIAGO NORIEGA ÁLVAREZ \\ El Bosque University, Electronics Engineering Department \\ Av. Cra 9 No. 131 A-02 Bogota, Colombia \\ HERNANDO LEÓN-RODRÍGUEZ \\ El Bosque University, Electronics Engineering and Bioengineering Department \\ Av. Cra 9 No. 131 A-02 Bogota, Colombia
}

Quadruped in comparison with the majority of others animals, it has the ability to access to any kind of environment where others living creatures or even humans can't access. Those bio-inspired attributes are taken into this project in order to design and develop a quadruped robot with the abilities to move in all kind of directions like ascend or descend, avoid obstacles, etc. Combining these skills of the quadruped animals with the ability of continuously monitoring the carbon dioxide, the results can be determinants. This paper presents the dynamic and kinematics model in addition with the measurement scheme of the carbon dioxide index, with the purpose of establish a mechanical sturdy device, which can be monitoring an important variable. As a result, we studied the movement of real animals, so we can define a suitable bio-mimetic model for our robot

\section{Introduction}

Recently, human has wanted to replicate all kind of movement of animal. This effort on doing it allowed humans to reproduce robots for certain task instead of risk their lives. This kind of biomimetic replication can be employed in land mines task and exploration task. [1] Another important application of these robots is the incursion in dangerous environments, like contaminated places, or hostile landmarks. In this order of ideas, we decided that the applicative background of the robot is the measurement of the concentration of carbon dioxide, which is an important variable that can generate negative impacts if no measurement (and regulation) control is applied. Besides, the human beings can be, easily, exposed to these gases without notice it, with mortal consequences. Since 90's researches begin to innovate the whole world with bio-inspired robot.[2] In 2008 bio-inspired robots became in an important instrument, with several ability of walking and climbing in all kind of surfaces. [3] [4]

Nowadays, the majority of quadruped robots have exceptional abilities and advanced material to be developed. Other approaches have been employed in 
their developed of quadruped robots as example parallel mechanism [5], soft materials [6] self-folding [7], Origami [7], printable robots and so on.

Nevertheless, this paper presents a device based on quadruped robots with some basic features and different attributes and applications. See figure 1

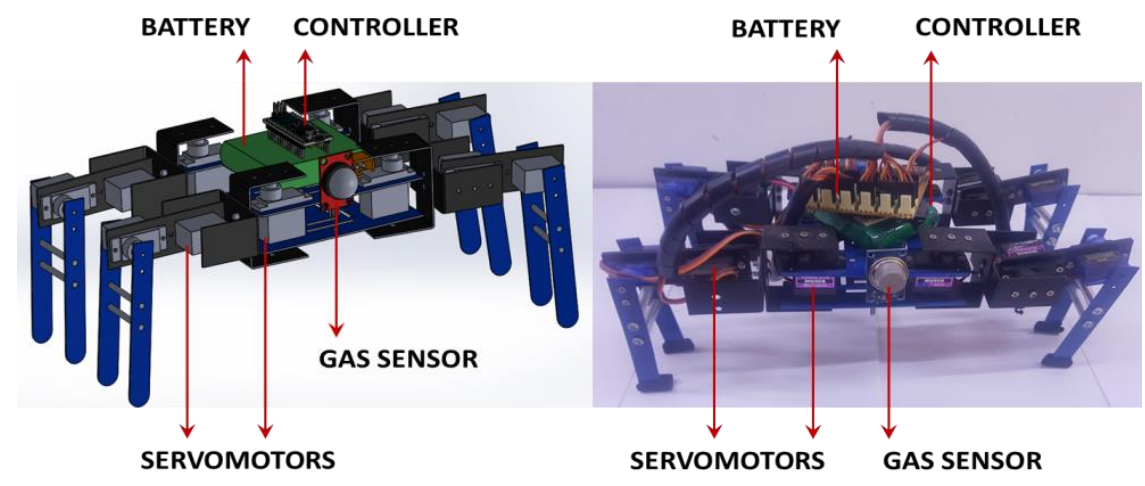

Figure 1: Quadruped robot design and prototype.

\section{The Bio inspired quadruped and motion analysis}

In order to accomplish this robot, we stablished how quadruped move and the algorithm behind this process. The majority of the quadrupeds move in a mammalian form, like a dog or a horse. Besides this, the spider has 8 limbs, so we couldn't use them as a direct source of inspiration. To solve this problem, we used a mixture of sources of inspiration; we had the quadrupedal animals (their movement and behavior). On the other hand, we had the anatomy of the spider. As a result, the anatomy or physic shape of the spider, and the movement of the quadruped animals in an arachnid way was used.

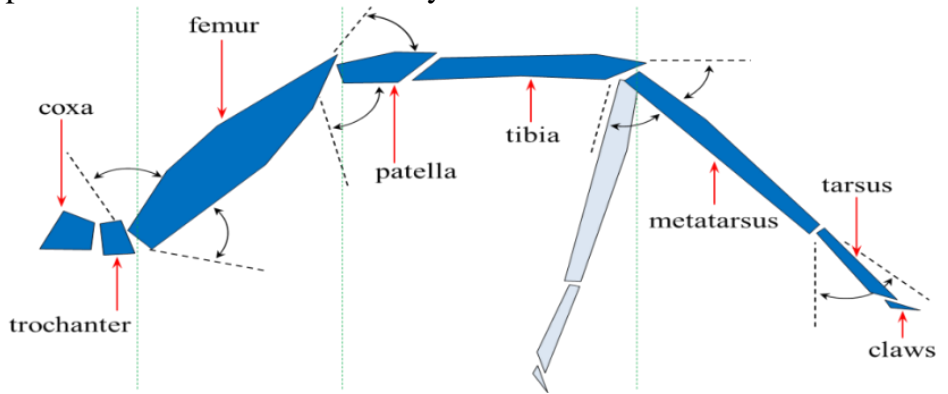

Figure 2: Spider's leg biomechanics

Initially, it's important to know that the spider has 7 parts by leg (figure 2). From the original anatomy of the spider, we suppress some components that we didn't need. The reason of this is that we wanted to simplify the whole system. 
Having said this, instead of using the Patella part, we linked the femur and the tibia by a direct joint. The metatarsus and the tibia were united as a single link or part. Similarly, we dismiss the tarsus. All of these changes were executed in the robot, but for the kinematics analysis we took into account the entirely system for a realistic approach.

One important aspect is the amplitude that has every part of the spider's leg. This means, for example, that the coxa has amplitude of 35 degrees while tibia has a mobility of 70 degrees. Also, every of the seven components of the limb, has a different axis of movement; for example, the trochanter has a movement in $\mathrm{X}-\mathrm{Y}$ axis, meanwhile the femur in $\mathrm{X}-\mathrm{Z}$ axis. This kind of association and motion is explained graphically in the figure 2 .

\section{Mathematical development}

As we mentioned previously, there are some constraints that we applied in the anatomic development. We applied these modifications in the mathematical development and we decided to involve all the possible variables, based on the following table to produces the most faithful model and prototype.

Table 1. Degrees and planes of work of each part of the quadruped robot.

\begin{tabular}{ccc}
\hline Parts & Movements (degrees) & Plane \\
\hline Coxa & 75 & Transversal \\
Femur & 140 & Sagittal \\
Tibia & 40 & Sagittal \\
\hline
\end{tabular}

\subsection{Direct Kinematics}

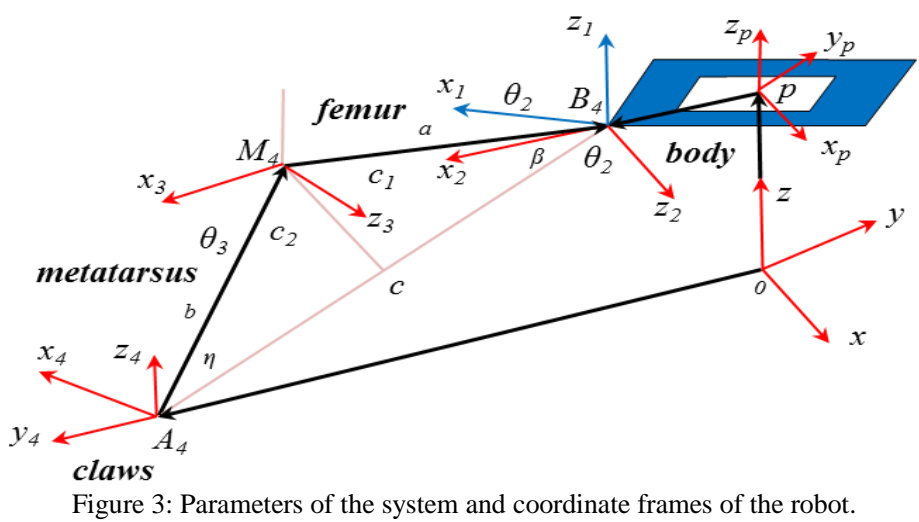

In order to study the direct kinematics of the robot was necessary, at the beginning; use the joint's variables of contact limbs and the position and 
orientation of the platform. All of this, based on fixed frame represented in figure 3. Is important to establish that the $A i$ vectors denote the end points of contact legs and the $B i$ vectors represent the connection points of the legs of the robot to the platform. Taking into account the figure 3 and knowing Ai vectors, which are the end points of contact legs, we can establish the next expression:

$$
r B i=r A i+\frac{r M i}{A i}+\frac{r B i}{M i}
$$

In this expression, $r B i$ and $r A i$ represent the position vector of $B i$. In the same way, we needed to determinate all the parameters of the system in a graphically mean. In the figure 3 these parameters can be observed.

One highly important aspect in our robot was the motion and the sequence that a quadruped robot must follow in order to walk correctly. This item is the quadruped walking system, which is illustrated in the figure 4.
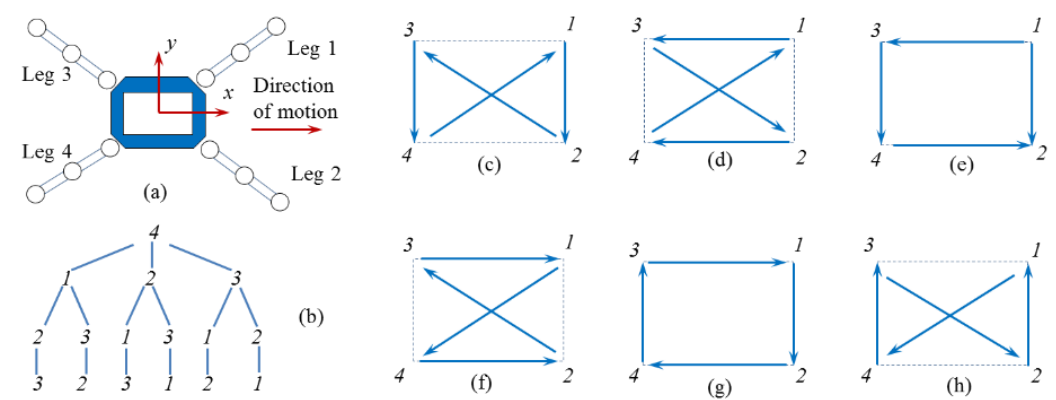

(b)
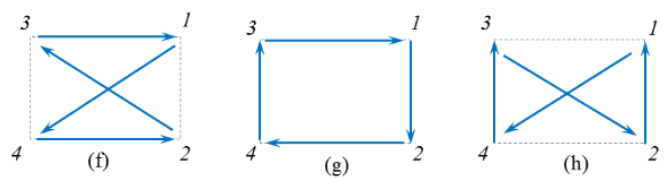

Figure 4: Sequence of quadruped walk

Suppose that the leg 1,2 and 3 are standing on the ground. According to relation (1) the location of points $B i$ versus the fixed coordinate system are determined and, as direction of $x$ axis of $\mathrm{P}$-coordinate system is direct to $B 3 B 1$ vector, is possible determine the direction of $\mathrm{x}$-axis unit vector:

$$
E x=\frac{B 3 B 1}{\llbracket B 3 B 1 \rrbracket}
$$

In the same way, we can determinate the vector $B 3 B 2$

$$
E m=\frac{B 3 B 2}{\llbracket B 3 B 2 \rrbracket}
$$

By having this information, we can determinate the direction of unit vector, normal to the platform plane. To do this, we first need to implement the cross product of the two previous vectors:

$$
E z=E m x E x
$$

In the same way, having the vectors $E x$ and $E z$, is possible to determine the Ey vector using the same method:

$$
E y=E x \times E z
$$


These three vectors are necessary because we can establish the matrix of the platform versus the fixed coordinate system with the next expression:

$$
{ }_{B}^{P} R=\left[\begin{array}{llll}
E x & E y & E z
\end{array}\right]
$$

In order to specify the origin of coordinate system, we can use the equation of the circle in this way:

$$
\begin{aligned}
& (X b 1-X p)^{2}+(Y b 1-Y p)^{2}+(Z b 1-Z p)^{2}=r^{2} \\
& (X b 2-X p)^{2}+(Y b 2-Y p)^{2}+(Z b 2-Z p)^{2}=r^{2} \\
& (X b 3-X p)^{2}+(Y b 3-Y p)^{2}+(Z b 3-Z p)^{2}=r^{2}
\end{aligned}
$$

If we solve the previously established equations system, we can determine the position of the body in the coordinate system.

\subsection{Platform velocity}

In order to establish the velocity of the robot's platform is necessary to determine the velocity and angular velocity of robot platform by using the position and velocity of the joint's variables. In order to specify the direct kinematics of platform velocity can use (10):

$$
\overrightarrow{O A_{l}}+\overrightarrow{A_{l} M_{l}}+\overrightarrow{M_{l} B_{l}}+\overrightarrow{B_{l} P}=\overrightarrow{O P}
$$

In the previous expression, $O A i$ represents a vector that was drawn from the fix coordinate origin to point " $\mathrm{A}$ " from leg No. i. It's possible to determinate the relation between velocity of the joint's variables and platform velocity by differentiating from (10). The result is (11):

$$
\overrightarrow{V p}=\stackrel{B \rightarrow T i b}{\omega_{i}} \times \overrightarrow{A_{i} M_{i}}+\stackrel{B \rightarrow F e m}{\omega_{i}} \times \overrightarrow{M_{i} B_{i}}+\stackrel{B \rightarrow P}{\omega} \times \overrightarrow{B_{i} P}
$$

In (11), the first and third element of the equality represents the absolute angular velocity of femur and tibia, of limb No. i respectively. If we take into account the symmetry of our robot, (11) this process can be used for the other three contact legs. By using the fifth element of (11), is possible to establish Vp. Based on figure 3:

$$
\begin{aligned}
\underset{1 \rightarrow T i b}{\omega_{i}} & =\theta_{1}{ }^{i} \vec{K}_{1}+\zeta^{i} \vec{K}_{2} \\
\underset{1 \rightarrow F e m}{\omega_{i}} & =\theta_{1}{ }^{i} \vec{K}_{1}+\zeta^{i} \vec{K}_{3}
\end{aligned}
$$

Regarding to the figure 3 :

$$
\begin{gathered}
\zeta=\frac{\pi}{2}-\theta_{2}-\theta_{3} \rightarrow \dot{\zeta}=\left(\dot{\theta_{2}}+\dot{\theta_{3}}\right) \\
\gamma=\frac{\pi}{2}-\theta_{2} \rightarrow \dot{\gamma}=-\dot{\theta_{2}}
\end{gathered}
$$

In expressions (12) and (13), the first factor in both of them, indicates the unit vector direct to z-axis of first coordinate frame of limb No. i. The relation 
between the unit vectors of different coordinate frames of each leg is determined in function of the figure 3 as follow:

$$
\begin{gathered}
\overrightarrow{l_{K_{3}}}=\overrightarrow{\overline{l_{K_{2}}}} \\
\overrightarrow{l_{K_{2}}}=-\sin \left(\theta_{1}\right) \overrightarrow{l_{I_{1}}}+\cos \left(\theta_{1}\right) \overrightarrow{l_{J_{1}}} \\
\overrightarrow{{\overrightarrow{l_{4}}}_{1}}=-\overrightarrow{l_{K_{3}}}
\end{gathered}
$$

Using the expressions from (12) to (18), we can determine the values of $\omega i$ as follows:

$$
\begin{gathered}
\stackrel{1 \rightarrow T i b}{\omega_{i}=\theta_{1} i} \vec{K}_{1}-\left(\theta_{2}+\theta_{3}\right)\left(-S\left(\theta_{1}\right) \vec{i}_{i_{1}}+C\left(\theta_{1}\right) \vec{i}_{j_{1}}\right) \\
\stackrel{1 \rightarrow F e m}{\omega_{i}}=\theta_{1}{ }_{i} \vec{K}_{1}-\theta_{2}\left(-S\left(\theta_{1}\right) \vec{i}_{i_{1}}+C\left(\theta_{1}\right) \vec{i}_{j_{1}}\right)
\end{gathered}
$$

In (19) and (20) the $S$ 's and the $C$ 's, means cosines and sines. In this case, for mathematical simplicity, we can express all the previous equations as rotational matrices as follows:

$$
\begin{gathered}
\underset{{ }_{i}^{B \rightarrow \text { Fem }}}{\omega_{i}}={ }_{B}^{P} R_{P} R_{i}{ }^{1 \rightarrow \text { Fem }} \omega_{i} \\
\begin{array}{c}
B \rightarrow \text { Fem } \\
\omega_{i}
\end{array}={ }_{B}^{1} R_{i}{ }^{1 \rightarrow \text { Fem }} \omega_{i} \\
\begin{array}{c}
B \rightarrow \text { Tib } \\
\omega_{i}
\end{array}={ }_{B}^{1} R_{i}{ }^{1 \rightarrow \text { Tib }} \quad \omega_{i}
\end{gathered}
$$

As we mentioned previously, $R$ represents the rotational matrix of platform relative to the fix coordinate frame. In this order $R 1 p$ is the rotation matrix of the first coordinate frame of limb No.i relative to P-coordinate frame system. This last rotational matrix is defined as follow:

$$
R=\left[\begin{array}{ccc}
\cos \left[(i-1) \frac{\Pi}{3}+\frac{\Pi}{6}\right] & -\sin \left[(i-1) \frac{\Pi}{3}+\frac{\Pi}{6}\right] & 0 \\
\sin \left[(i-1) \frac{\Pi}{3}+\frac{\Pi}{6}\right] & \cos \left[(i-1) \frac{\Pi}{3}+\frac{\Pi}{6}\right] & 0 \\
0 & 0 & 1
\end{array}\right]
$$

\subsection{Direct kinematics of non-contact leg}

Direct kinematics of position for a non-contact limb is similar to the direct kinematics for a serial robot. As shown in Figure 3 can write:

$$
\begin{gathered}
O A i=O P+P B i+B i M i+M i A i \\
P B i={ }_{B}^{P} R P B i \\
B i M i={ }_{B}^{P} R{ }_{P}^{1} R B i M i \\
M i A i={ }_{B}^{P} R{ }_{P}^{1} R M i A i
\end{gathered}
$$

Based on the previous expressions, $P B i$ can be establishing as follows:

$$
P B i=\left[\begin{array}{c}
r \cos \left(\frac{\Pi}{6}+(i-1) \frac{\Pi}{6}\right) \\
r \sin \left(\frac{\Pi}{6}+(i-1) \frac{\Pi}{6}\right) \\
0
\end{array}\right]
$$


As we did with the contact legs, we wanted to determine the velocity of the non-contact limbs, so the procedure is similar. We first need to differentiate (25) as follows:

$$
\overrightarrow{V_{A i}}=\overrightarrow{V p}+\stackrel{B \rightarrow P}{\omega} \times \overrightarrow{B_{i} P}+\stackrel{B \rightarrow F e m}{\omega_{i}} \times \overrightarrow{B_{i} M_{i}}+\stackrel{B \rightarrow T i b}{\omega} \times \overrightarrow{M_{i} A_{i}}
$$

Using $V p$ and $\omega$, that were derived from (11):

$$
\begin{gathered}
\stackrel{B \rightarrow F e m}{\omega}=\dot{\theta}_{i}{ }^{i} \underline{K}_{1}+\dot{\theta}_{2}{ }^{i} \vec{K}_{2}+\stackrel{B \rightarrow P}{\omega} \\
\stackrel{B \rightarrow T i b}{\omega}=\theta_{3}{ }^{i} \vec{K}_{3}+\theta_{1}{ }^{i} \vec{K}_{1}+\theta_{2}{ }^{i} \vec{K}_{2}+\stackrel{B \rightarrow P}{\omega}
\end{gathered}
$$

From (30) to (32) we can determine the velocity of end points of noncontact legs; as a result, these values can be specified.

\section{Software and Carbon Dioxide Reading Sensor}

The robot control was based in arduino controller and Bluetooth communication system, sending and receiving routine commands from mobile device. We used an Arduino-Nano as the controller for the full platform control and communication system. For motion, 12 servo-actuators were set, 3 for each leg with a torque of $2.2 \mathrm{~kg} . \mathrm{cm}$. These servo-motors are attached directly as a joint of each link-leg. The supply voltage and current for the robot was a battery package of $4.8 \mathrm{~V}$ and $3000 \mathrm{~mA}$ with around power of $7.5 \mathrm{~W}$ approx.

Regarding to the reading of the carbon dioxide index, an embedded circuit capable of monitoring various types of gases was implemented.
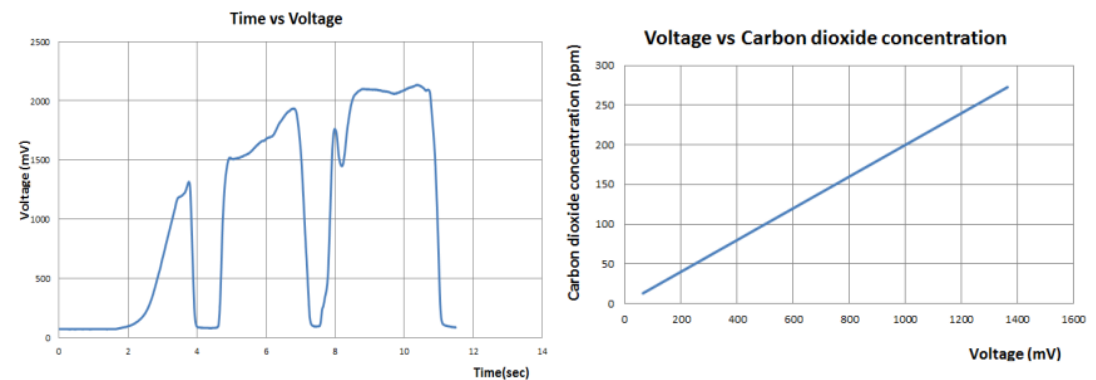

Figure 5: left: Response profile MQ4, time vs voltage; right: Voltage vs Carbon dioxide.

The figure 5-left shows the output voltage of the sensor depending of the gas concentration reading by itself. The result was obtained by adapting the gas source next to the sensor; meanwhile the gas concentration was increasing.

Figure 5-right shows the relation of output voltage with the carbon dioxide concentration; in this way, the characterization of the sensor was adapted through a linear regression model, obtaining accurate results. An extremely important aspect to mention is the fact that this issue is still being evaluated and 
treated, with the purpose of implementing the sensor that best shapes to the context and conditioning with the purpose of achieving the best results.

\section{Conclusions}

The project has achieved systematically the design, development and control of a quadruped walking robot. The mathematical model helped out the modeling of the motion's behavior of the robot. The robot has 12 DOF in total, 3 DOF for each leg, controlled by an Arduino Nano via a remote mobile device. The movement has been analyzed with biomimetic inspirations take from the spiders. The gas sensor MQ4 was an excellent first approach to the gas sensing technology because it allowed characterizing the behavior of the gas. Additionally, these results will serve us as a foundation in future research.

\section{References}

1. Zhao Tang, Peng Qi, Jian Dai, (2017) "Mechanism design of a biomimetic quadruped robot", Industrial Robot: An International Journal, Vol. 44 Issue: 4, doi: 10.1108/IR-11-2016-0310

2. S. Shoval, E. Rimon and A. Shapiro, "Design of a spider-like robot for motion with quasi-static force constraints," Proceedings 1999 IEEE International Conference on Robotics and Automation, Detroit, MI, 1999, pp. 1377-1383 vol.2. doi: 10.1109/ROBOT.1999.772553

3. Yi Lu, Keke Zhou, Nijia Ye, Design and kinemics/dynamics analysis of a novel climbing robot with tri-planar limbs for remanufacturing, Journal of Mechanical, Science and Technology, March 2017, Volume 31, Issue 3, pp 1427-1436

4. Claudio Semini, HyQ - Design and Development of a Hydraulically Actuated Quadruped Robot, A thesis submitted for the degree of Doctor of Philosophy (Ph.D.) April 2010.

5. Hongbo Wang, Zhengyan Qi, Guiling Xu, Fengfeng Xi, Guoqing Hu and Zhen Huang, Kinematics Analysis and Motion Simulation of a Quadruped Walking Robot with Parallel Leg Mechanism, The Open Mechanical Engineering Journal, 2010, 4, 77-85

6. Garabini M., Santina C.D., Bianchi M., Catalano M., Grioli G., Bicchi A. (2017) Soft Robots that Mimic the Neuromusculoskeletal System. In: Ibáñez J., González-Vargas J., Azorín J., Akay M., Pons J. (eds) Converging Clinical and Engineering Research on Neurorehabilitation II. Biosystems \& Biorobotics, vol 15. Springer, Cham.

7. Cagdas D. Onal, Member, IEEE, Michael T. Tolley, Member, IEEE, Robert J. Wood, Member, IEEE, and Daniela Rus, Fellow, IEEE; OrigamiInspired Printed Robots; IEEE/ASME TRANSACTIONS ON MECHATRONICS, VOL. 20, NO. 5, OCTOBER 2015 